\title{
CARD GAME ANALYSIS FOR FAST MULTI-CRITERIA DECISION MAKING
}

\author{
Valentino Sangiorgio $^{1, *}$, Beatrice Di Pierro ${ }^{1}$, Michele Roccotelli $^{2}$ \\ AND Bartolomeo Silvestri ${ }^{2}$
}

\begin{abstract}
Multi-criteria decision methods are very popular in decision-making for many application fields thanks to their versatility and ability to involve qualitative and quantitative data in the analysis. On the other hand, the many of these techniques requires time consuming analysis and the involvement of expert users in the decision-making process. In this paper, a novel method named Card Game Analysis (CGA) is proposed to perform Multi-criteria Decision Making (MCDM) analysis. Such methodology uses a set of cards and a suitable game procedure to perform the analysis, allowing to determine indirectly tabulated weights of involved parameters (criteria, sub-criteria, alternatives, etc.) and obtain the ranking of priorities. The CGA is defined following the footsteps of the "structuring of the problem", typical of the Analytic Hierarchy Process (AHP), and takes inspiration from an evaluation procedure presented in the related literature. In particular, the CGA is composed by the following steps: (1) structuring of the problem, (2) card setting, (3) card game weights evaluation, (4) synthesis of priorities. Finally, a validation through a comparison with the AHP procedure is performed by exploiting 25 case studies regarding the desirability of the enabling technologies of the Industry 4.0 for a set of companies in the Adriatic Ionian area.
\end{abstract}

Mathematics Subject Classification. 91B06, 90B50.

Received November 22, 2019. Accepted April 7, 2021.

\section{INTRODUCTION}

Decision-making is the action that humans perform every day in different fields of real life and is a very important part of human daily activities both in private life and professional work. In this context, some decisions can be taken relatively easily, especially if the consequences of wrong decisions are not critical, while others can be very complex and have important effects. Real-life decision problems are complex problems that involve several conflicting criteria that should be taken into account simultaneously, in order to achieve a reasonable decision [21].

In this framework and in the field of operational research, management and decision science, multi-criteria decision-making (MCDM) approaches have been widely studied from the academic world. In particular, several MCDM methods have been proposed and applied to decision problems in different areas, dealing with the design of computational and mathematical tools for supporting the subjective evaluation of performance criteria by

Keywords. Decision science, multi-criteria decision methods, card game analysis.

1 DICATECH, Politecnico di Bari, Via Edoardo Orabona 4, Bari, Italy.

2 DEI, Politecnico di Bari, Via Edoardo Orabona 4, Bari, Italy.

*Corresponding author: valentino.sangiorgio@poliba.it 
decision-makers $[21,26]$. In this context, MCDM methods have been designed to classify alternatives in a small number of categories, choose preferred alternative, and rank alternatives in a subjective preference order. More generally, MCDM methods help the decision maker to clarify their preferences in cases where there are conflicting criteria [24]. In particular, MCDM methods allow decomposing complex problems into their basic components in order to analyze each aspect individually on the basis of different criteria. After each component is weighted and judged, all the problem parts can be reassembled to recompose the whole problem puzzle and give it to the decision maker. The majority of MCDM methods are based on discrete alternatives, described by a set of criteria. The values of criteria can be determined as an ordinal or cardinal information and each information can be determined exactly or with a fuzzy logic. Considering that the daily life decisions are based on several criteria, a decision can be made by assigning weights to each criterion by means of group of experts. To this aim, it is essential to select the structure of the decision problem and clearly evaluate all the criteria. Furthermore, all the alternatives should have common criteria so to lead the DM to better and more informed decisions.

There are different classifications of MCDM methods that have been proposed in the related literature. According to the nature of the information that can be cardinal, ordinal and mixed scales, it is possible to distinguish among quantitative (or hard), qualitative and mixed (or soft) MCDM methods. For instance, MCDM quantitative methods are the weighted sum $[27,42]$ and the elimination and choice expressing reality (ELECTRE) $[13,19]$. Among the qualitative methods there are the REGIME methods [23], the lexicographic technique [41] and the multidimensional scaling analysis [14].

The mixed methods are the most diffused MCDM approaches such as the Evaluation of Mixed Data (EVAMIX) method [15], the Technique for Order of Preference by Similarity to Ideal Solution (TOPSIS) $[8,38]$, the Analytic Hierarchy Process (AHP) [17,26,32,33], the Analytic Network Process (ANP) [11,34], the multiobjective optimization by ratio analysis (MOORA) [10], and multiple objective optimization on the basis of ratio analysis plus full multiplicative form (MULTIMOORA) [6,25], the weighted aggregated sum product assessment (WASPAS) $[3,12]$.

A second classification can be done based on the level of compensation that is allowed by the method. In this context, the MCDM methods can be compensatory (such as Multi-Attribute Utility Theory [40], AHP and the ANP), partially-compensatory (such as Preference Ranking Organization Method for Enrichment Evaluation [7,39], the REGIME and the Novel Approach to Imprecise Assessment and Decision Environments [28]) and non-compensatory (for instance Dominance method [9]).

In particular, all these MCDM methods are applied in many areas such as: construction and project management, energy, environment, sustainability, supply chain, ICT, safety and risk management, operation research and soft computing and other fields $[1,4,26,43]$.

There are some issues that affect some of the aforementioned MCDM approaches such as the choice of the aggregation procedure, the linear trade-offs among factors (dependent on the problem structure), the zero as a natural scale of factors, and the achievement of subjective judgments before the decision-making process [26]. Other important limitations that are common to all the MCDM methods are the following: (1) the MCDM process is time-consuming that collide with the few time available by the users; (2) the user needs to be expert in the specific field of analysis; (3) the user needs to keep high concentration in all the process phases in order to obtain good and consistent results. Because of these issues, it is rare to obtain reliable results in complex and extensive problems.

In particular, also the AHP method, that is one of the most adopted MCDM tools by decision makers and researchers in different areas [16,30], is affected by those limitations. The AHP is a method of "measurement through pairwise comparisons and relies on the judgments of experts to derive priority scales" [33]. The AHP methodology is based on a hierarchical structure able to hierarchically measure a multiplicity of factors of a complex process, allowing to simply recompose the parts in a whole. In order to have a good decision, the decision maker should define the problem, the needs and goals of the decision, the criteria and sub-criteria to evaluate the alternatives, the actions to be taken and the interested stakeholders [33]. The AHP method is basically based on three consecutive phases [16,33]: (1) Define the problem, the decision criteria and hierarchy; (2) Build matrices to determine a set of pairwise comparison and calculate the elements weights; (3) Sensitivity 
analysis. Even if the AHP method is largely used, its main limitation, like for other cited methods, is that the selection of criteria and the determination of the weights basically depends on the expertise knowledge. Therefore, people involved in the AHP process should be expert in the field of analysis and dedicate time to efficiently accomplish all the process tasks and ensure the success of the methodology application.

In this paper, a novel approach, named Card Game Analysis (CGA), is proposed in order to overcome some of the cited limitations affecting the most common MCDM methods. The CGA falls in the set of mixed and compensatory methods. It is based on a multi-step procedure that allows achieving similar results of AHP but by means of the card game approach that is more user-friendly and less time consuming. In particular, the CGA is defined on the basis of the problem structuring of the AHP methodology and takes inspiration from the Simos-Roy-Figueira method (SRF) [18] for the weight's evaluation. In particular, the weight evaluation is reworked by a new equation and a consistency check for fast and effective decision making. The CGA represents a technique devoted to allowing any DM (not essentially familiar with multi-criteria decision tools) to explain the way in which he/she wants to rank the different criteria in a given context.

Unlike the methods from which it is inspired, the proposed CGA overcomes some drawbacks by reworking the positive aspects of both AHP and SRF techniques in a new process. In particular, this work proposes a very simple procedure, using a set of cards to determine indirectly the weights of parameters based on the following steps: (1) structuring the problem; (2) setting the cards; (3) evaluating the card game weights, (4) synthesis of priorities. After the problem has been structured, the user, which carries out the weighting, uses a specific and suitable designed card sets: the name of each criterion is written on each card together with other (complementary) information. Moreover, the user receives a set of white cards with the same size. Therefore, users can start the card game by sorting the cards based on the importance of the represented criteria and uses the blank cards to increase the distance between two consecutive cards. Local weights are then extracted from the ranking of the cards and suitable consistency tests are performed.

The novelties of the proposed method are listed in the following items.

(i) Compared to AHP, the CGA method is completely based on a visual, fast and intuitive procedure in a card play approach. For these reasons, the CGA method is especially suitable for problems involving non-expert users who can carry out the procedure successfully thanks to the useful information stored in the cards.

(ii) Compared to SRF card method, the card game procedure can be applied several times to obtain all the weights defined in the hierarchical structure of the problem. In addition, a novel algorithm for weights evaluation is proposed, based on the aggregation principles of local weights of the AHP.

(iii) Compared with other MCDM, the proposed approach is particularly effective in order to evaluate human behaviors, priorities and beliefs. In addition, the CGA can be applied in decision processes where an expert is not necessary in the process.

Furthermore, thanks to the simple and quick steps of the decision process, the card game can be played in short time, since the necessary information is included in the cards.

In order to validate the proposed method, the paper performs a comparison with the AHP by carrying out the application to 25 case studies.

The paper is organized as follows. Section 2 describes the CGA approach specifying the step-based procedure; Section 3 presents the comparison with the AHP methodology in several case studies for CGA validation while Section 4 discusses the limitations of the method and the future perspectives. Finally, Section 5 draws the conclusions.

\section{THE CARD GAME ANALYSIS}

\subsection{Overview of the method: the four steps of the CGA}

In this section the novel procedure of the CGA is described on the basis of the following 4-steps: (1) structuring the problem (CGA Step 1); (2) card setting (CGA Step 2); (3) card game weights evaluation (CGA Step 3), (4) synthesis of priorities (CGA Step 4). 


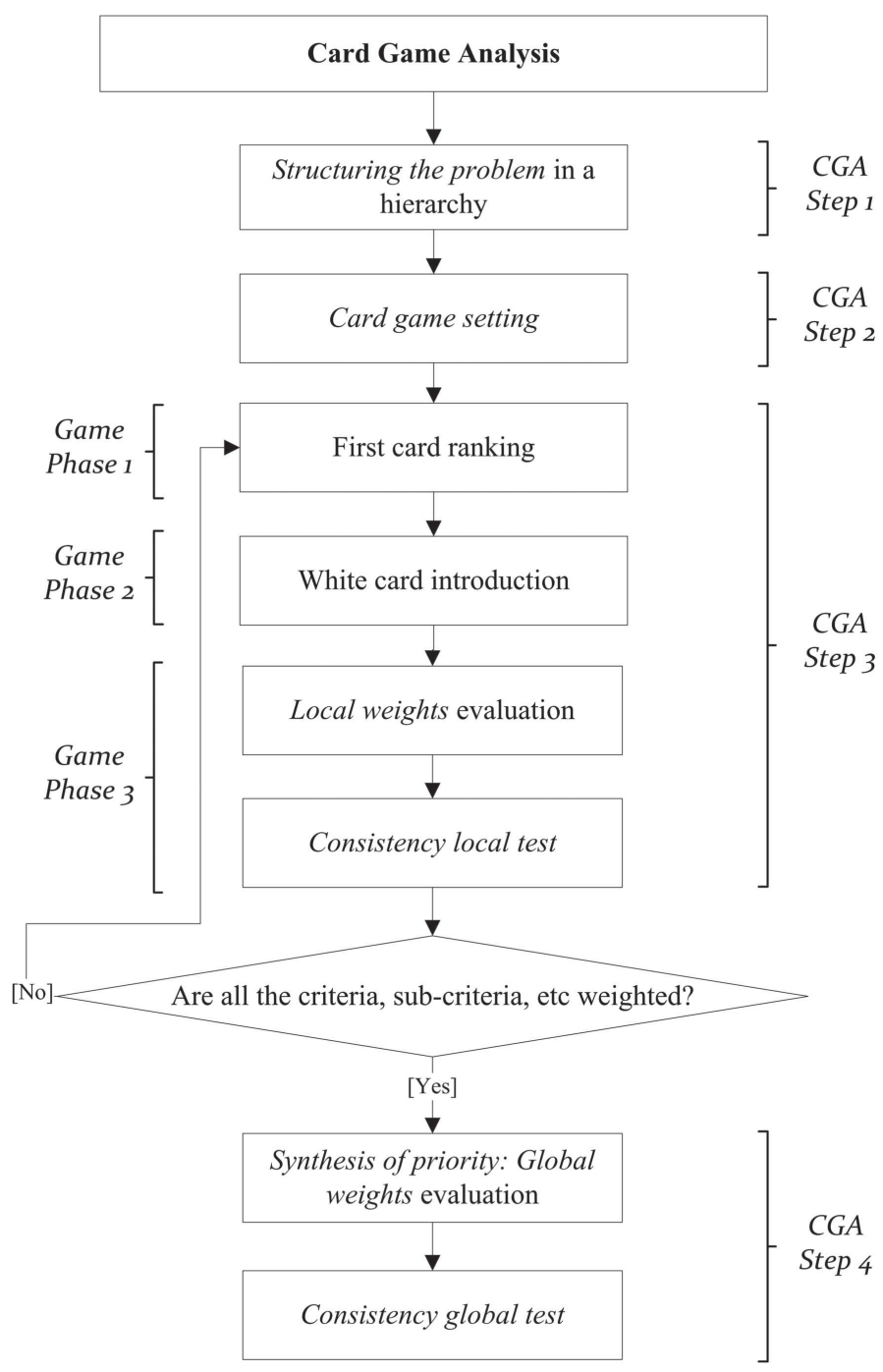

Figure 1. The flowchart of the CGA procedure.

In particular, Step 1 follows in the footsteps of the AHP [33] and is devoted to decomposing and structuring the problem in a flowchart to obtain a complete overview of the involved parameters, which are classified as criteria, sub criteria and alternatives. In Step 2, the card game is set by following a specific procedure to create the cards and a suitable local consistency test is defined. Step 3 regards the application of the card game in three game phases to perform multi criteria evaluation: firstly (Game Phase 1) the users employ the cards to perform a first ranking; secondly (Game Phase 2) the ranking and the importance of the cards are expressed; thirdly (Game Phase 3) the local weights are extracted and a local consistency test is performed to verify the coherence of the results. Finally, in Step 4, if all parameters are weighted, the synthesis of priorities is performed to obtain the global weights and a global consistency test can be performed. If the parameters are not all verified by the tests, the procedure goes back to Game Phase 1. In the rare case that the DM is unable to reach consistency numerous times, he/she can be considered not reliable, similarly to the AHP, and therefore not considered in the analysis results [35]. 


\begin{tabular}{l}
\hline Level 1 \\
\hline Level 2 \\
\hline \\
Level 3 \\
\hline
\end{tabular}

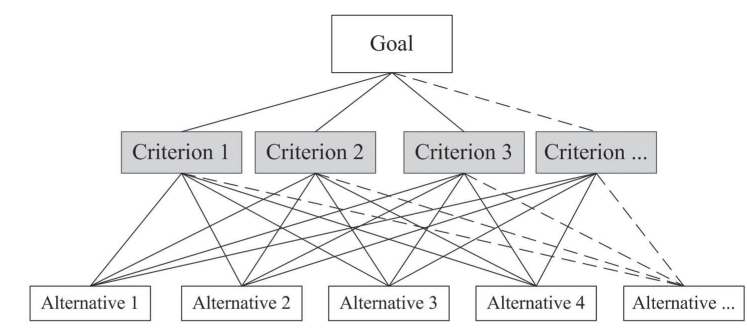

Level ...

FiguRE 2. The generic structure of the problem according to a hierarchical flowchart.

Figure 1 shows an overview of the method and illustrates the fundamental steps and phases in a flowchart.

\subsection{CGA step 1: Definition of goal, criteria, sub criteria and alternatives}

The CGA Step 1 comprises the identification of the goal and the structuring of the problem according to a hierarchical scheme composed by different levels. This step provides a detailed, simple and systematic decomposition of the problem parameters into its basic components. This scheme represents the goal of the analytical process, the involved criteria, sub-criteria, alternatives (that are generally called parameters in this paper) that are classified in different levels, to have a complete description of the considered phenomenon. It is worth noting that there are no-limits to the number of levels to the hierarchical scheme (Fig. 2).

\subsection{CGA step 2: The card setting}

The CGA Step 2 is of basic importance for the CGA and in this phase the cards are proposed. The number of necessary cards is equal to the total number of criteria, sub-criteria and alternatives structured in the flowchart of CGA Step 1. In particular, a card is designed for each parameter involved in the analysis (i.e., criteria, sub criteria or alternative): the name of each parameter is written on each card together with additional (complementary) information.

In particular, every single card needs to enclose the following information to be effective: (i) the name of the parameter that refers to the problem structure of CGA Step 1; (ii) a representative photo or scheme; (iii) a synthetic description; (iv) quantitative data, which can be typological, functional, characteristic or economic data; (v) advantages; (vi) disadvantages; (vii) other information. Figure 3 shows the generic card layout.

\subsection{CGA step 3: Card game for weights evaluation}

Once all the cards have been created, it is possible to play the card game to analyze every single aspect of the decision problem. Following the hierarchical flowchart, a set of games is performed for every level until all criteria, sub criteria and alternatives are weighted. In the following, the card game with relative weight extraction and consistency test is explained.

\subsubsection{Card game}

Let us assume that a set of cards is defined to evaluate a set of criteria, sub criteria or alternatives. Since the method is based on human judgments, every user can start the game following the described three game phases. Such procedure allows identifying perception, priorities and beliefs of the involved users (or DMs) about the structured problem.

In Game Phase 1 (Fig. 4), the user is asked to rank the cards from the less important to the most important according to his/her perception. So, the user orders the cards by assigning to them an ascending importance (parameter): the first card in the rank is the less important and the last card is the most important. If the user 


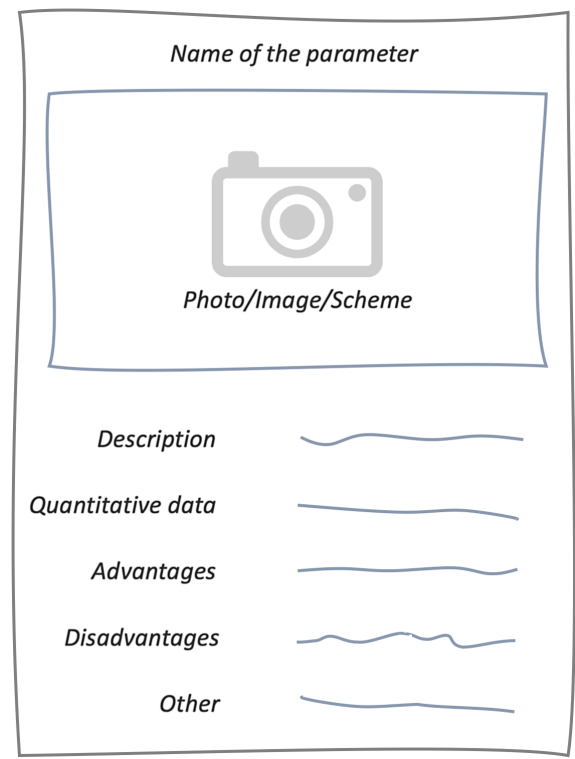

Figure 3. The generic Card layout.

\section{Most important}

\section{Less important}
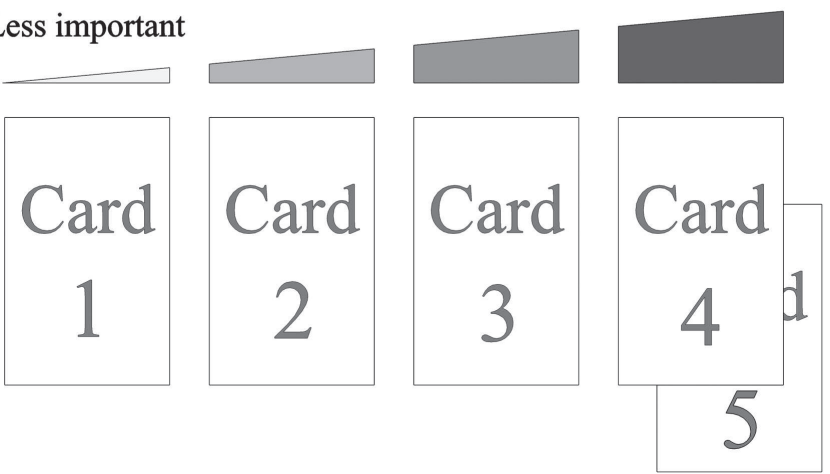

Figure 4. Example of Game Phase 1: first card ranking from less important to most important.

decides that some parameters have the same importance (i.e., the same weight), the user can assign the same position to a subset of cards. Consequently, the output of Game Phase 1 is a complete pre-order basis on the considered parameters.

In Game Phase 2, the user can decide about the importance of two successive cards (or subsets of cards) in the ranking. Indeed, users can introduce white cards between two consecutive cards (or subsets of cards) in order to increase the differences in the ranking: the absence of white cards between two consecutive cards means small difference. The greater the number of used white cards is, the greater is the ranking difference between the two cards. The Game Phase 2 goes to an end when with the assignment of a rank, both to the card, white cards, and subsets of cards (Fig. 5). 


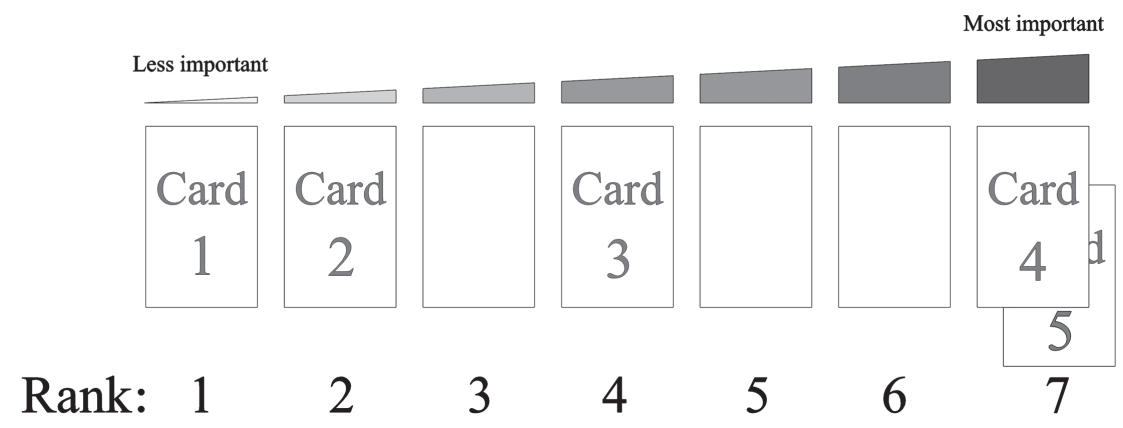

Figure 5. Example of Game Phase 2: use of white cards and rank.

\subsubsection{Weights determination}

In the Game Phase 3 it is possible to derive weights from the result of Game phase 2.

Let us assume that a set $N=\{p \mid p=1, \ldots, n\}$ of $n$ parameters are analyzed through the card game and let us denote $C=\left\{c_{p} \mid p=1, \ldots, n\right\}$ the set of cards $c_{p}$ where $c_{p}$ is associated to the $p$ th parameter for $p=1, \ldots, n$. Moreover, we define the set $C w=\left\{c w_{q} \mid q=n+1, \ldots, m\right\}$ (with $m>n$ ) of white cards and the set $C T=C \cup C w$ includes the cards associated to parameters and the white cards. In addition, a rank $r_{q} \in \mathbb{N}$ (for $q=1, \ldots, m$ ) is assigned to each card in $C T$ during $C G$ phase 2 , where $\mathbb{N}$ is the set of natural numbers. The local weight $v_{p} \in \mathbb{R}^{+}$associated to each card $c_{p} \in C$, and then to each parameter $p$, is computed by the following formula:

$$
v_{p}=\frac{r_{p}}{\sum_{q=1}^{m} r_{q}}, \text { for } \quad p=1, \ldots, n
$$

where $\mathbb{R}^{+}$is the set of real positive numbers and it holds $v_{p} \in[0,1]$ with $\sum_{p=1}^{n} v_{p}=1$.

\subsubsection{Local consistency test}

The consistency local test is performed in order to verify if the user is aware of the choices made during the card game and to verify that the resulting weights are coherent. The test is used to verify that the DM correctly reflected his/her perception with the game of cards. Being based exclusively on the DM perception, there is no need for particular expertise to carry out this verification. In particular, the user randomly draws two cards and performs a card pair comparison expressing numerically how much one card is more important than the other. Let us assume that the user extracts the cards $c_{p}$ and $c_{q}$ and assigns a value $k_{p, q} \in \mathbb{R}^{+}$to this pair comparison.

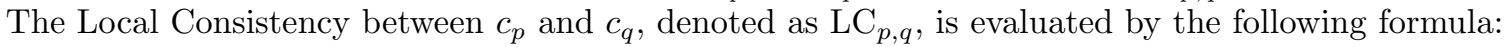

$$
\mathrm{LC}_{p, q}=\left|\frac{k_{p, q}-\left(v_{p} / v_{q}\right)}{v_{p} / v_{q}}\right|, \quad \text { for } \quad p, q=1, \ldots, \mathrm{n}, p \neq q .
$$

On the basis of several empirical studies, it is possible to assume that the values $0 \leq \mathrm{LC}_{p, q} \leq 0.3$ are acceptable [32]. It is worth noting that this first local consistency test does not ensure that the final result is reliable, but it indicates whether the user has a good perception of the importance of the analyzed parameters.

\subsection{CGA step 4: Synthesis of priorities}

In the final CGA step 4, the synthesis of priorities (or weights aggregation) is performed to determine the rankings and the global weights for each alternative by following the approaches used in related literature for the MCDMs.

To this aim, the weights of each criterion and sub-criterion are combined with the weights of the alternatives in order to obtain the global weights. The global weights are typically organized in tabulated weights useful to complete the multi criteria analysis and obtain chart to get a visual overview of the results. 
In the related literature there are many equations to perform the weights aggregation and the use of a specific equation depends from the investigated problem [35-37]. This work uses the widespread weights aggregation presented in the related literature: the global weights are obtained by multiplying each criteria weight by the alternative weight and totaling the results for each alternative [22].

\subsubsection{Global consistency test}

In order to obtain a complete feedback about the coherence of the analysis, a global consistency test can be set and applied to conclude the Game Phase 4. Unlike the consistency local test carries out a quantitative verification (Game Phase 3), a qualitative test is proposed to verify the global weights. Note that this subsection presents a generic description of the global consistency test that has to be customized in each particular application of the CGA.

More precisely, the test is composed by three steps: (i) a suitable and concise questionnaire is set in order to obtain qualitative information regarding the user preferences; (ii) the user answers the questionnaire after performing the card game phases and before becoming aware of results (global weights, ranking and charts); (iii) a qualitative evaluation is performed to assess any incoherencies between the card game results and the questionnaires answers.

A qualitative classification can be assigned to the CGA user: low coherence, medium coherence or good coherence.

It is worth noting that the rationality of this test is based on the idea that the user after the card game is played can reach a good knowledge of the problem, to this aim he/she should be able to express resolutely his/her preferences through the questionnaire.

\section{VALidATION THROUGH CASE STUDies AND AHP COMPARISON}

In order to validate the proposed CGA, in this section a suitable comparison with the AHP, based on a set of case studies, is proposed. The AHP is the most appropriate methodology to perform the comparison, indeed the CGA Step 1 and Step 4 follows the footsteps of the methodology of [33]. Because of this, the two methods provide similar results that can be easily compared as the global weights having the same normalization. In this subsection, the case studies about the evaluation of the potentiality of the Industry 4.0 technologies in the Adriatic-Ionian area are described. In particular, firstly the CGA is applied, secondly the AHP procedure is briefly described and applied, and finally the results of the two methods are compared to validate the new procedure.

\subsection{The case studies}

The application field concerns the fourth industrial revolution that in recent years became the key point of research and innovation fields. To this aim an excellent knowledge of industrial change and enabling technologies of Industry 4.0 became fundamental in the manufacturing sector in order to have a complete overview of the potentiality and desirability of enabling technologies and to plan education and training governance models.

To this aim the choice of case studies regards the actual topic of the desirability of the enabling technologies of the Industry 4.0 for a set of companies in the Adriatic Ionian area (Fig. 6). In particular, the persons responsible of the management and the technological innovation of 25 companies are selected as the users of the MCDMs.

\subsection{The Card Game Analysis application}

The CGA step 1 is applied to structure the problem for the potentiality's evaluation of the main Industry 4.0 enabling technologies.

To this aim the following six criteria $i$ (with $i=1, \ldots, 6)$ are defined to consider the advantages and disadvantages of the technologies: (1) Professional skills required for using the technology; (2) Short-term economic benefit; (3) Long-term economic benefit; (4) Initial costs to implement the technology; (5) Operating costs; (6) 


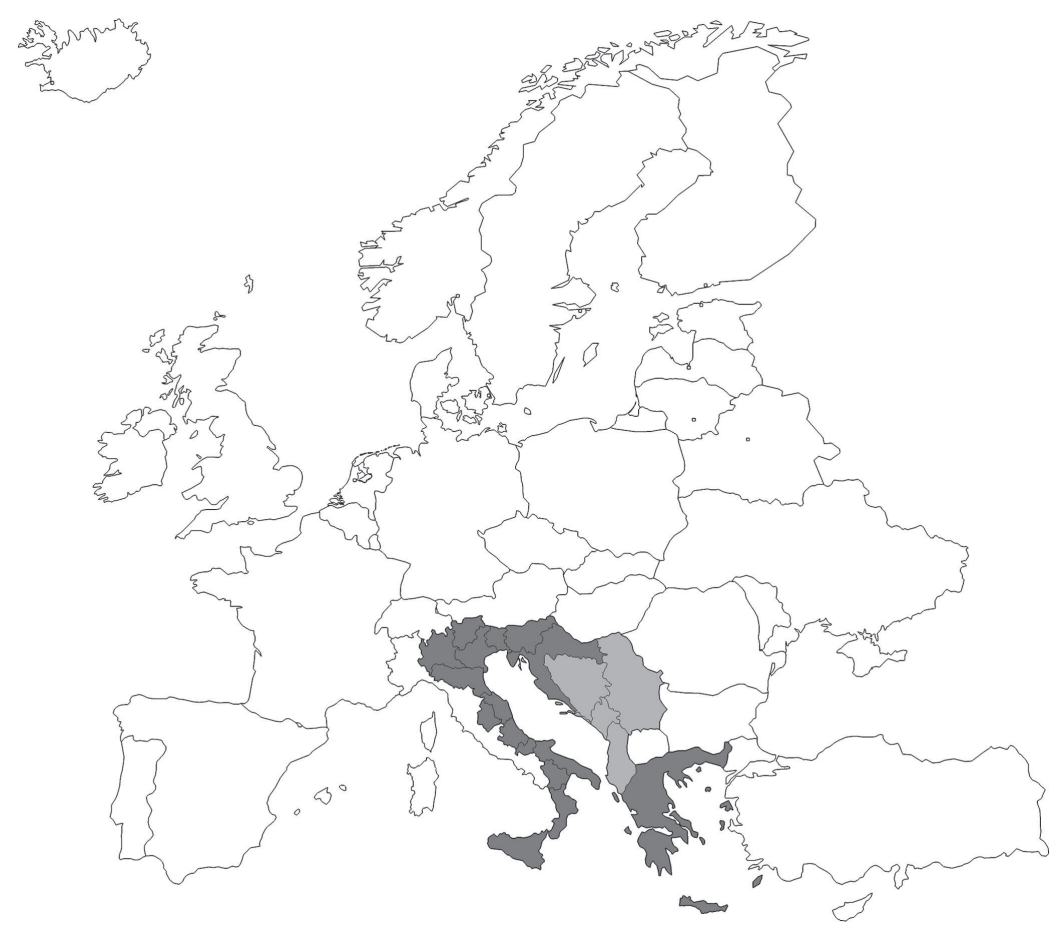

Figure 6. Adriatic-Ionian area.

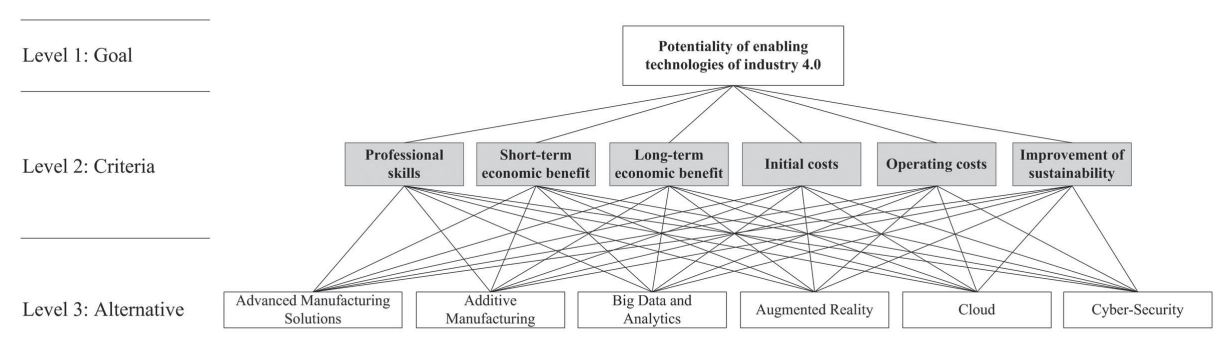

Figure 7. The structure of the problem: potentiality of the enabling technologies of Industry 4.0.

and Improvement of sustainability related with the use of the technology. In addition, six main enabling technologies are defined as alternative $j$ (with $j=1, \ldots, 6$ ) of the problem: (i) Advanced Manufacturing Solutions (AS); (ii) Augmented Reality (AR); (iii) Cloud Computing (CC); (iv) Additive Manufacturing (AM); (v) Big Data and Analytics (BD); (vi) Cyber-security (CS).

Figure 7 shows the problem structure regarding the potentialities of the Enabling technologies of Industry 4.0.

Once the problem is defined, the CGA step 2 is applied and the cards $c_{j}$ associated with alternative $j$ (for $j=1, \ldots, 6)$ are specified. In particular, cards regarding the enabling technologies are showed: (i) the name of the criterion or alternative, (ii) a representative photo or scheme of the technology; (iii) a synthetic description of the technology; (iv) cost of implementation; (v) advantages, (vi) and required skills to use the technology.

Figure 8 shows the cards of the enabling technologies defined as alternatives. 


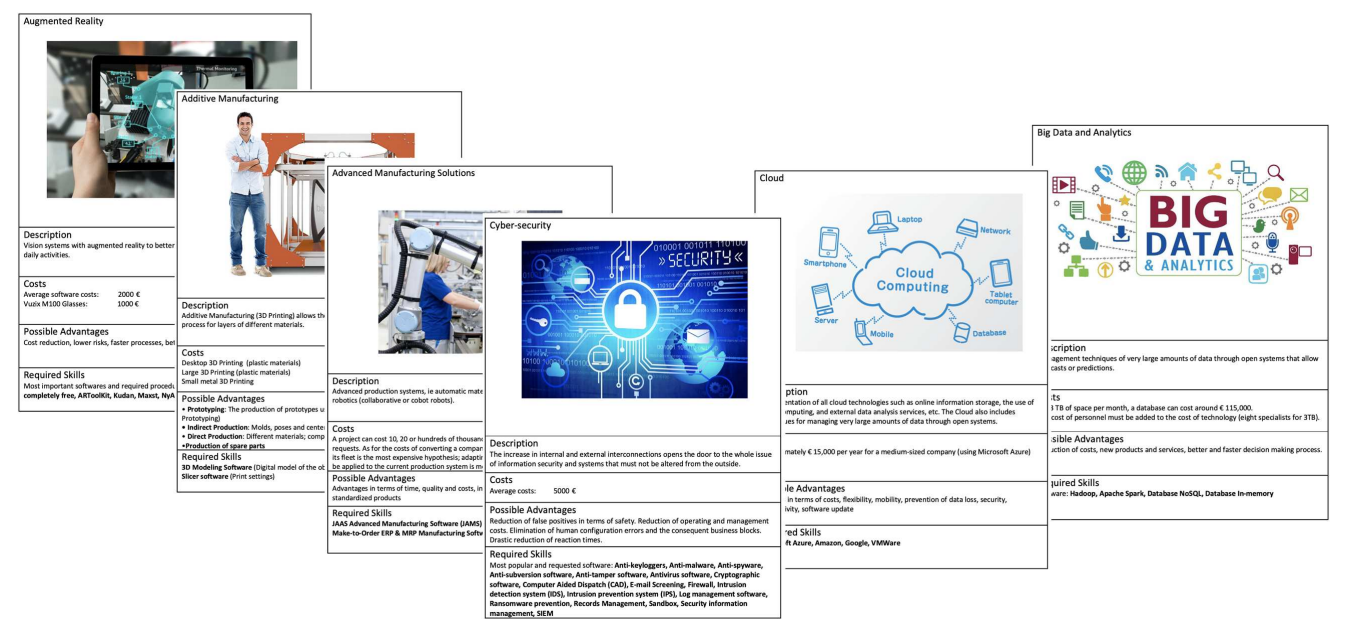

Figure 8. Cards of enabling technologies of Industry 4.0.

In CGA step 3 every criterion and alternative are analyzed in order to weight the involved parameters. Let us define the set of criteria $N_{1}=\{i \mid i=1, \ldots, 6\}$ and the set of alternatives $N_{2}=\{j \mid j=1, \ldots, 6\}$. The criteria and alternatives local weights are defined as follows:

- $v_{i}$ is the local weight associated with the $i$ th criterion $\forall i \in N_{1}$;

- $w_{i, j}$ is the local weight associated with the $j$ th alternative related to the $i$ th criterion, for $\forall i \in N_{1}, \forall j \in N_{2}$.

Starting from user 1 of the first considered company, the CGA step 3 allows the evaluation of local weights: six games are used to identify the local weights $w_{i, j}$ and one to identify the weight of criteria $v_{i}$. In particular, local weights $v_{i}$ are computed on the basis of equation (2.1) by considering $p=i$. In addition, local weights $w_{i, j}$ are computed by reworking equation (2.1) as follows:

$$
w_{i, j}=\frac{r_{j}}{\sum_{q=1}^{m} r_{q}}, \forall j \in N_{2}, \forall i \in N_{1} .
$$

To provide an example, the first game is performed by user 1 that in the Game Phase 1 considers the enabling technologies (all alternatives $j \in N_{2}$ ) in relation to the professional skills $(i=1$ ). The user orders the technologies from the one that requires fewer professional skills than the others. Subsequently, in Game Phase 2 a set of white cards are used to increase the differences between two consecutive technologies. Figure 9 shows an example of the CGA in the end of the Game Phase 2.

In the Game Phase 3 the weights $w_{1, j}$ are determined by equation (3.1). Figure 10 shows the rank assigned to every card and the extraction of weights $w_{1, j}$ by equation (3.1).

In addition, the local consistency check is performed. For instance, according to phase 3 the user extracts the card of Additive manufacturing $\left(c_{2}\right)$ and Big data and analytics $\left(c_{3}\right)$ and assign a value of $k_{3,2}=3$ : i.e., in the user perception Big data and analytics has a weight 3 times greater than Additive manufacturing. By reworking equation (2.2), to the extracted cards, it is possible to verify the Local Consistency.

$$
\mathrm{LC}_{3,2}=\left|\frac{3-\left(w_{1,3} / w_{1,2}\right)}{w_{1,3} / w_{1,2}}\right|
$$

$0 \leq \mathrm{LC}_{3,2}=0.068 \leq 0.3$ and it is possible to consider that user 1 has consistently applied the card game phase. Analogously, the card game three phases are performed for all the criteria and alternatives. In this way, all values of $v_{i}$ and $w_{i, j} \forall j \in N_{2}, \forall i \in N_{1}$ are determined. 


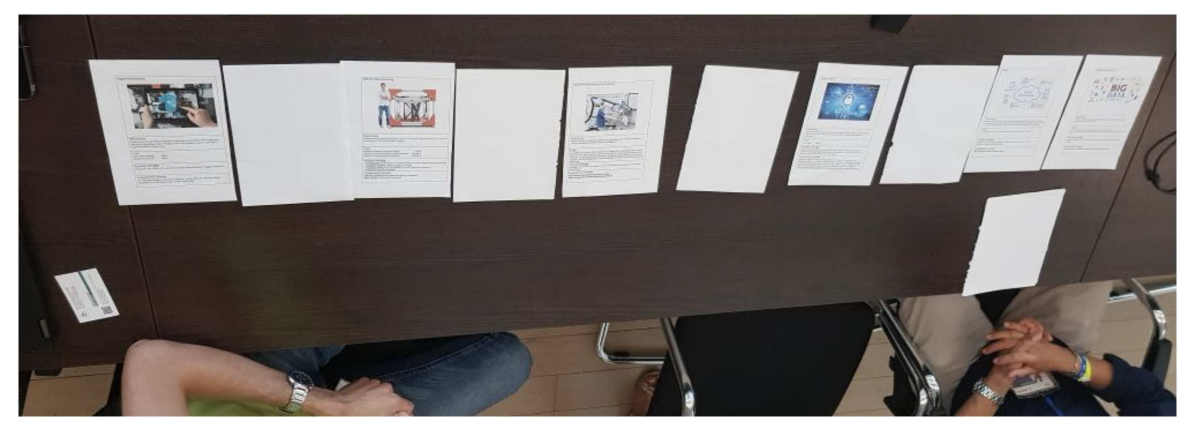

FIGURE 9. User 1 performs the card game on enabling technologies (alternatives) in relation with professional skills (first criterion).

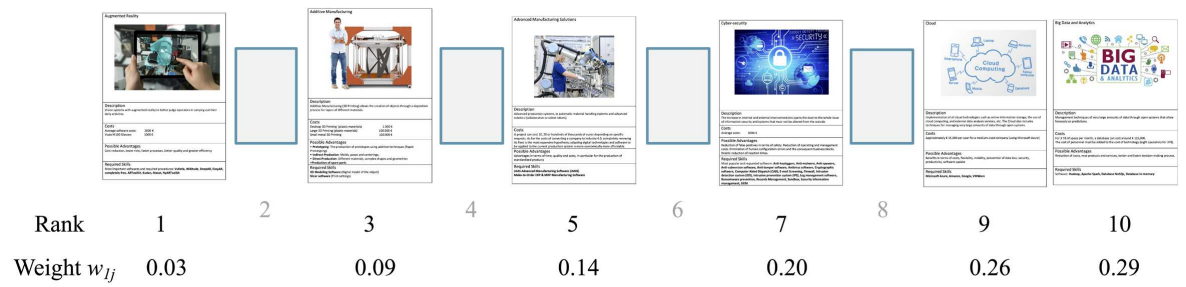

FiguRE 10. Rank and local weights $w_{1, j}$ of alternatives for the first criterion.

After obtaining all the weights of criteria and alternatives related to each criterion, it is possible to evaluate the global weights $w_{j}^{\prime}$ (CGA step 4) representing the effective preferences of the user 1 with regard to the enabling technologies. In particular, the following equation is used to obtain the synthesis of the priority.

$$
w_{j}^{\prime}=\sum_{i=1}^{6} v_{i} \times w_{i, j}, \forall j \in N_{2}
$$

The resulting values of $w_{j}^{\prime}$ of user 1 are showed in Figure 11 through a pie chart expressing the global weight as a percentage.

Finally, the consistency global test is set and applied. In order to validate the obtained weights, a set of questionnaires is organized to understand if the analyzed company just employ or intends to employ some of the enabling technologies (in Fig. 11 if company 1 uses the enabling technology is indicated with "yes").

In particular, if the technologies with the highest weights (it means most desirable technologies according to the company needs) result also those that are just applied (or will be applied shortly) the global weights are coherent. Figure 11 shows the resulting global weights $w_{j}^{\prime}$ expressed in percentage and the technologies used in company 1. It is worth noting how in this case the global consistency is perfectly respected since the technologies with the greatest potential are also the most used in company 1. A qualitative classification of the consistency is expressed as Good coherence.

The procedure is applied to 25 users and companies.

\subsection{The AHP evaluation}

In this subsection, in order to compare the results obtained by the CGA method in the 25 companies, the AHP is applied to the same case studies of Section 3.2, by following the well-known Saaty 3-steps Method [33]: (i) structuring the problem in a hierarchy, (ii) weight evaluation, (iii) global weight evaluation. 


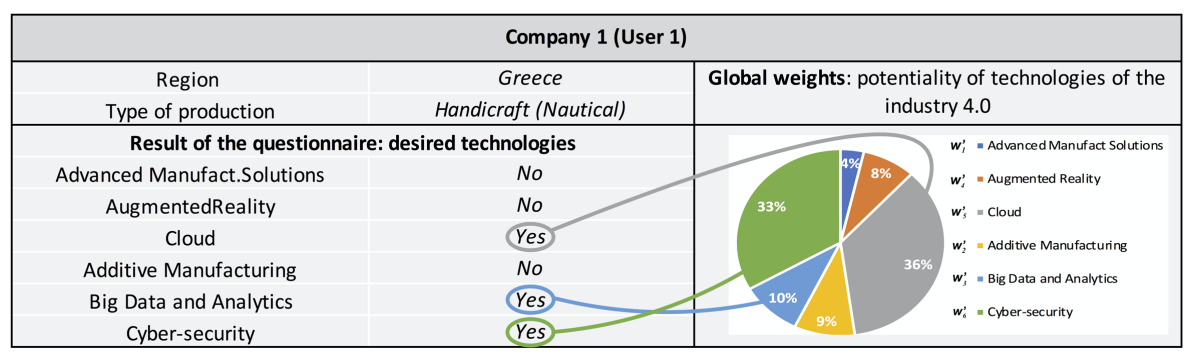

FIGURE 11. Global Weights expressed in percentage and global consistency check.

\begin{tabular}{ccccc}
\hline$A$ & 1 & 2 & $\ldots$ & $n$ \\
\hline 1 & 1.0 & $a_{1,2}$ & $\ldots$ & $a_{1, n}$ \\
2 & $1 / a_{2,1}$ & 1.0 & $\ldots$ & $a_{2, n}$ \\
$\ldots$ & $\ldots$ & $\ldots$ & 1.0 & $\ldots$ \\
$n$ & $1 / a_{1, n}$ & $1 / a_{2, n}$ & $\ldots$ & 1.0 \\
\hline
\end{tabular}

Figure 12. The generic matrix of judgments $A$.

TABLE 1. Fundamental scale of Saaty [32].

\begin{tabular}{ll}
\hline \hline$a_{i j}$ & Verbal scale \\
\hline$a_{i j}=1$ & Equal importance \\
$a_{i j}=3$ & Moderate importance of one over another \\
$a_{i j}=5$ & Strong importance \\
$a_{i j}=7$ & Very strong importance \\
$a_{i j}=9$ & Extreme importance \\
$1.5-4-6-8$ & Intermediate value \\
$1 / 9,1 / 8, \cdots, 1 / 2$ & The reciprocal expresses an opposite judgement \\
\hline
\end{tabular}

The first AHP step comprises the identification of the goal and the structuring of the problem according to a hierarchical flowchart as already described for the CGA method. In the presented case study, the step consists of determining the structure of the problem analogously to the CGA step 1 (see Fig. 7): potentialities of the enabling technologies of Industry 4.0.

The second AHP step of local weight evaluation is the main step of the method and provides the weights of every criterion and alternative. Such operation allows analyzing each characteristic of the phenomenon and examining qualitative and quantitative parameters. In particular, a $n \times n$ judgments matrix $A$ (Fig. 12) is defined by considering the $n$ criteria of comparison (i.e., criteria, sub-criteria or alternatives): the upper diagonal element $a_{i j}>0$ is determined by comparing the $i$ th element with the $j$ th one by taking into account the fundamental scale of Saaty (Tab. 1).

At this point the ratio scale priority vectors are derived by determining the principal eigenvalues and the corresponding eigenvectors of the positive reciprocal matrices as follows:

$$
A w=\lambda_{\max } w
$$


TABLE 2. Random consistency index of Noble [29].

\begin{tabular}{llllllllllllllll}
\hline \hline$n$ & 1 & 2 & 3 & 4 & 5 & 6 & 7 & 8 & 9 & 10 & 11 & 12 & 13 & 14 & 15 \\
\hline RI & 0 & 0 & 0.49 & 0.82 & 1.03 & 1.16 & 1.25 & 1.31 & 1.36 & 1.39 & 1.42 & 1.44 & 1.46 & 1.48 & 1.49 \\
\hline
\end{tabular}

\begin{tabular}{c|cccccccc}
\hline$A_{1}$ & $A S$ & $A R$ & $C C$ & $A M$ & $B D$ & $C S$ & $C R$ & $w_{1 j}^{A H P}$ \\
\hline Advanced Manufacturing Solutions $(A S)$ & 1.0 & 5.0 & 0.5 & 1.0 & 0.5 & 0.7 & & 0.13 \\
Augmented Reality (AR) & 0.2 & 1.0 & 0.1 & 0.2 & 0.1 & 0.1 & & 0.03 \\
Cloud Computing (CC) & 2.0 & 9.0 & 1.0 & 1.5 & 0.8 & 1.3 & 0.002 & 0.24 \\
Additive Manufacturing (AM) & 1.0 & 5.0 & 0.7 & 1.0 & 0.5 & 0.7 & & 0.14 \\
Big Data and Analytics (BD) & 2.0 & 9.0 & 1.3 & 2.0 & 1.0 & 1.5 & & 0.27 \\
Cyber-security (CS) & 1.4 & 8.0 & 0.8 & 1.4 & 0.7 & 1.0 & & 0.19 \\
\hline
\end{tabular}

Figure 13. Judgment Matrix $A_{1}$, weights, and CR obtained for the professional skills criterion.

where $\lambda_{\max }$ is the principal eigenvalue and $w$ is the corresponding eigenvector. Moreover, in order to check the coherence of the obtained weights, Saaty defines the consistency index CI. Such index increases proportionally with the inconsistency of the paired comparisons:

$$
\mathrm{CI}=\frac{\lambda_{\max }-n}{n-1} .
$$

Operationally, to verify the coherence of the matrix and the result with a parameter that is independent of the matrix order, the Consistency Ratio (CR) [32] is determined. More precisely, the value of CR is computed as the ratio between $\mathrm{CI}$ and random consistency index $\mathrm{RI}(n)$. In particular, $\mathrm{RI}(n)$ is determined by considering a large number of positive reciprocal matrices of order $n$, whose entries are randomly chosen in the set $\{1,2, \ldots, 15\}$. The following relationship holds:

$$
\mathrm{CR}=\frac{\mathrm{CI}}{\mathrm{RI}(n)} .
$$

In order to consider a large number of alternatives, we assume $\operatorname{RI}(n)$ for $1 \leq n \leq 15$ and the values of Noble and Sanchez [29] are used (Tab. 2) [2]. Furthermore, on the basis of the empirical studies of Saaty [32], the value of $\mathrm{CR}<0.10$ is acceptable.

Referring to the considered case study, the second AHP step provides the local weights that are defined as follows:

- $v_{i}^{\text {AHP }}$ is the local weight associated with each $i$ th criterion $\forall i \in N_{1}$;

- $w_{i j}^{\mathrm{AHP}}$ is the local weight associated with the alternative $j$ th $\forall j \in N_{2}$ related to the $i$ criterion $\forall i \in N_{1}$.

In the same way of the CGA, the procedure starts from user 1 of the first analyzed company and the second AHP step allows the evaluation of local weights: six matrices are evaluated to derive the local weights $w_{i j}^{\mathrm{AHP}}$ and one to identify the weight of criteria $v_{i}^{\mathrm{AHP}}$.

To this aim a pairwise comparisons of the enabling technologies in relation with the professional skills is carried out to achieve the judgment matrix $A_{1}$. Solving the eigenvector problem of equation (3.4) for matrix $A_{1}$ the local weights are obtained.

The resulting matrix satisfies the $\mathrm{CR}$ requirement $\mathrm{CR}<0.1$ and derives consistent weights $w_{1 j}^{\mathrm{AHP}}$ (see Fig. 13). Analogously, the AHP second step is performed for all the criteria and alternatives. In this way, all values of $v_{i}$ and $w_{i, j} \forall j \in N_{2}, \forall i \in N_{1}$ are determined.

Finally, the third AHP step, i.e., the global weight evaluation, is performed to evaluate the global weights $w_{1 j}^{\prime A H P}$ representing the effective preferences of the user 1 with regard to the enabling technologies. In particular, 


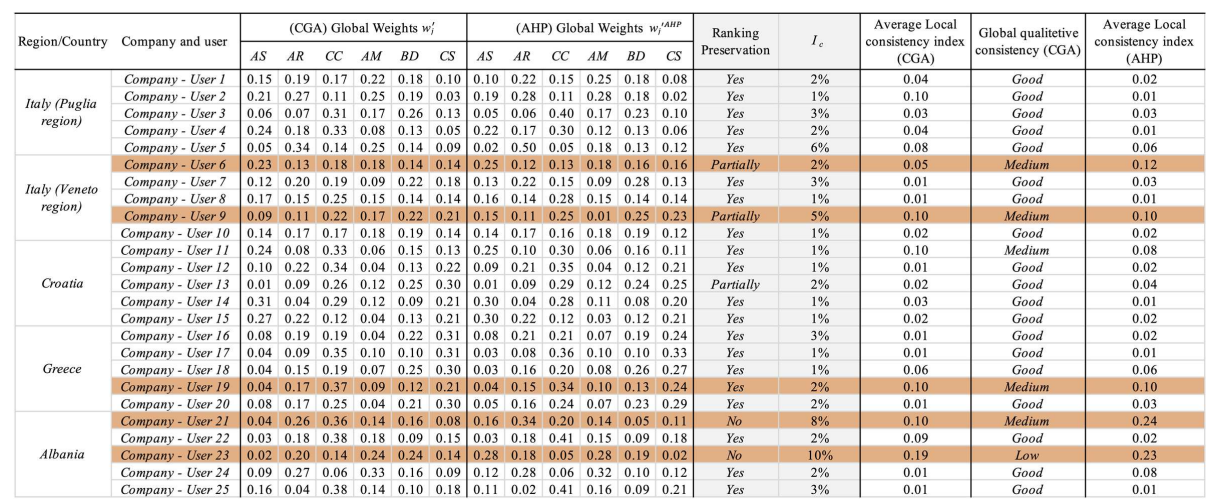

FiguRE 14. Comparison results: global weights, ranking, index of comparison and consistency tests.

the following equation is used to obtain the synthesis of priority:

$$
w_{j}^{\mathrm{AHP}}=\sum_{i=1}^{6} v_{i}^{\mathrm{AHP}} \times w_{i j}^{\mathrm{AHP}} .
$$

\subsection{Results and validation}

To validate the proposed CGA approach, it is necessary to compare the resulting global weights with the outputs of the AHP approach. The approach to compare the two methodologies considers two aspects: (i) the ranking preservation, that is one of the most important results of a MCDM; (ii) the differences of the global weights, that are fundamental to obtain a reliable numerical result in many typologies of decision problems.

The ranking preservation of the results of the two methods can be judged as follows: (i) respected, when the ranking obtained by the two methods is the same; (ii) partially respected, if there are only two criteria with different ranking; (iii) not respected if more than two criteria are different in the comparison.

Moreover, in order to compare the global weights of the two methods, an additional Index of comparison $\left(I_{c}\right)$ is defined as the average of the differences between the local weights of the two methodologies and is expressed by the following equation:

$$
I_{c}=\frac{\sum_{i=1}^{6}\left|w_{j}^{\mathrm{AHP}}-w_{j}^{\prime}\right|}{6} .
$$

Figure 14 shows a set of data resulting from the 25 case studies: the global weights of CGA and AHP for every alternative (enabling technology), the two aspects of the comparison, and the consistency test results of both methods.

It is possible to point out the following consideration by comparing the two methods:

- The rows in red (User $6 ; 9 ; 19 ; 21 ; 23$ ) show the results that do not respect the AHP consistency test. Therefore, they cannot be acceptable and are excluded from other comparisons. On the other hand, it is possible to compare the CGA consistency check with these rows. The data show an excellent behavior of the new consistency tests and both the Average Local consistency index in CGA and the Global qualitative consistency in CGA indicate a low coherence of the user in accordance with the AHP.

- The ranking preservation column shows that (for consistent results) the $95 \%$ of the ranking of the two methods is perfectly equal.

- The Index of comparison $I_{c}$ points out that the differences of the weights of the two methods are less than $6 \%$ for all the consistent results and the average of the differences is about $3 \%$ (considering all the 25 users).

In addition, other useful data are reported in order to compare the two methodologies: 


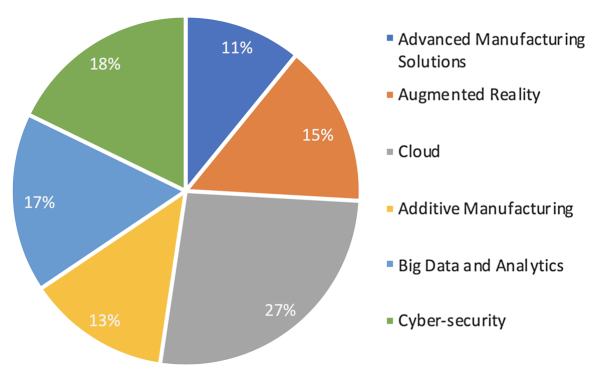

FIgURE 15. Global desirability of enabling technologies in Adriatic Ionian area expressed in percentage.

- The time to perform the CGA (7 card games to obtain all global weights) are 20 min on average, on the contrary the AHP requires $1 \mathrm{~h}$ on average. This is a very important parameter to consider the level of attention of the user that remains high in all the phases of the CGA.

- The users find much easier to assign a judgment in the CGA thanks to the effectiveness of the visual (card-based) procedure and the useful information about criteria and alternatives stored in the card.

- The result and validation test shows that in this type of problem the CGA has the same reliability as the AHP, in addition the comparison result confirms a greater simplicity and speed of application of the proposed method.

In order to show an example of the results obtained by these procedures, the proposed case studies are discussed in the following.

The obtained analysis of the industrial changes provides a complete overview of the desirability of enabling technologies of the fourth industrial revolution in the manufacturing sector. The results allow obtaining a structured ranking of the current technological framework in the Adriatic and Ionian area. The global desirability of enabling technologies in Adriatic Ionian is expressed in Figure 15.

These results will be useful in the future research in order to realize a platform to provide a smart learning model. This strategy will be implemented and validated through local pilot actions, encompassing an effective industrial education and training for innovation by enhancing the University-Industry cooperation. The results of this study can be the lay for the designing of knowledge, competence and skills training/learning hub involving universities and training organizations, companies and authorities.

\section{Limitations of the Method}

The proposed CGA method does not overcome all the limitations of other standard MCDM methodologies. In particular, some typical limitations of the MCDM, observable also in the described procedure are listed as follows: (1) the linear trade-offs among factors (dependent on the structure of the problem), (2) the zero as a natural scale of factors, and (3) the accomplishment of subjective judgments earlier in the decision-making process. It is worth noting that the same described limitations are identified in related literature also for the AHP method [5]. In addition, the CGA method is actually not able to specify the maximum number of cards in the same game to perform effective results (in the AHP the limit of the human mind for simultaneous comparison is equal $7 \pm 2$ ). Unlike other MCDMs which are applied for more than fifty years in different fields of application, the CGA will need many years of applications to state that there are not some specific fields in which some critical issues could arise to define some limitations in the number of simultaneous cards in a game. 


\section{Concluding Remarks}

This paper proposes a novel approach named Card Game Analysis (CGA) based on a multi-step procedure inspired by the AHP and exploiting a visual based comparison of parameters, introduced for the first time in the Simos-Roy-Figueira method [18]. This ambitious research project is carried out in three phases: (i) the definition of the novel CGA 4-steps; (ii) the application and test of the CGA 4-steps by 25 users to analyze complex decision problem of the "desirability of enabling technologies of Industry 4.0 in Adriatic Ionian"; (iii) the validation and comparison of the CGA with the AHP method in order to assess the effectiveness of the new approach.

The resulting method is based on a very simple procedure, using a set of cards, allowing to determine indirectly numerical values for weights. Moreover, the card set is suitably designed to be helpful in the understanding of the parameters involved in the problem. In addition, the method is provided with two consistency tests: (i) the first one is a quantitative test and allows verifying the coherence of the local weights; (ii) the second one is a qualitative test and is devoted to verifying any incoherencies of the global weights. The peculiarities of the CGA method allow overcoming some of the drawbacks of the most common MCDM, such as: (i) the difficulty of applying a MCDM even by users who do not have a complete knowledge of the problem; (ii) the difficulty to carry out the complete method in a short time to allow a high concentration of the user in all the steps; (iii) the possibility of verifying local and global consistency.

In addition, the results of this work show how the differences of the CGA and AHP methods are less than $6 \%$ demonstrating a good effectiveness of the proposed approach.

In conclusion, this method opens up new possibilities to apply MCDM in a simpler, faster and more accessible way, in order to be able to carry out analysis with an ever-increasing number of users.

Future research will focus on a complete analysis of the possibility of applying the method in different MCDA application fields. In addition, in order to increase the applicability, the CGA will be implemented in DSS and smart devices, in order to obtain a fast and customized method applicable on a large scale.

\section{REFERENCES}

[1] H. Allaoui, Y. Guo, A. Choudhary and J. Bloemhof, Sustainable agro-food supply chain design using two-stage hybrid multiobjective decision-making approach. Comput. Oper. Res. 89 (2018) 369-384.

[2] J.A. Alonso and M.T. Lamata, Consistency in the analytic hierarchy process: a new approach. Int. J. Uncertainty, Fuzziness Knowledge Based Syst. 14 (2006) 445-459.

[3] J. Antucheviciene and J. Saparauskas, MCDM methods WASPAS and MULTIMOORA: Verification of robustness of methods when assessing alternative solutions. Econ. Comput. Econ. Cybern. Stud. Res. 47 (2013) 5-20.

[4] C. Araz, P.M. Ozfirat and I. Ozkarahan, An integrated multicriteria decision-making methodology for outsourcing management. Comput. Oper. Res. 34 (2007) 3738-3756.

[5] P. Arroyo, I.D. Tommelein and G. Ballard, Comparing AHP and CBA as decision methods to resolve the choosing problem in detailed design. J. Constr. Eng. Manage. 141 (2014) 04014063.

[6] T. Baležentis and S. Zeng, Group multi-criteria decision making based upon interval-valued fuzzy numbers: an extension of the MULTIMOORA method. Expert Syst. App. 40 (2013) 543-550.

[7] M. Behzadian, R.B. Kazemzadeh, A. Albadvi and M. Aghdasi, PROMETHEE: a comprehensive literature review on methodologies and applications. Eur. J. Oper. Res. 200 (2010) 198-215.

[8] M. Behzadian, S. Khanmohammadi Otaghsara, M. Yazdani and J. Ignatius, A state-of the-art survey of TOPSIS applications. Expert Syst. App. 39 (2012) 13051-13069.

[9] D. Bonvin and D.A. Mellichamp, A generalized structural dominance method for the analysis of large-scale systems. Int. J. Control 35 (1982) 807-827.

[10] W.K.M. Brauers, Multi-objective seaport planning by MOORA decision making. Ann. Oper. Res. 206 (2013) 39-58.

[11] W.K. Brauers, Optimization Methods for a Stakeholder Society, A Revolution in Economic Thinking By Multi-Objective Optimization. In: Vol. 342 of Nonconvex Optimization and Its Applications. Kluwer Academic Publishers and Springer, Boston-Dordrecht-London (2004).

[12] S. Chakraborty and E.K. Zavadskas, Applications of WASPAS method in manufacturing decision making. Informatica 25 (2014) $1-20$.

[13] S. Corrente, S. Greco and R. Słowiński, Multiple criteria hierarchy process with ELECTRE and PROMETHEE. Omega 41 (2013) 820-846.

[14] T.F. Cox and M.A. Cox, Multidimensional Scaling. Chapman and Hall/CRC (2000). 
[15] V.P. Darji and R.V. Rao, Application of AHP/EVAMIX method for decision making in the industrial environment. Am. J. Oper. Res. 3 (2013) 542.

[16] R. de FSM Russo, R. Camanho, Criteria in AHP: a systematic review of literature. Proc. Comput. Sci. 55 (2015) $1123-1132$.

[17] A. Emrouznejad and M. Marra, The state of the art development of AHP (1979-2017): a literature review with a social network analysis. Int. J. Prod. Res. 55 (2017) 6653-6675.

[18] J. Figueira and B. Roy, Determining the weights of criteria in the ELECTRE type methods with a revised Simos' procedure. Eur. J. Oper. Res. 139 (2002) 317-326.

[19] J.R. Figueira, V. Mousseau and B. Roy, ELECTRE methods. In: Multiple Criteria Decision Analysis: State of the Art Surveys. Springer, New York, NY (2005) 133-153.

[20] J.R. Figueira, S. Greco, B. Roy and R. Słowiński, An overview of ELECTRE methods and their recent extensions. J. MultiCriteria Decis. Anal. 20 (2013) 61-85.

[21] K. Govindan and M.B. Jepsen, ELECTRE: a comprehensive literature review on methodologies and applications. Eur. J. Oper. Res. 250 (2016) 1-29.

[22] P.T. Harker and L.G. Vargas, The theory of ratio scale estimation: Saaty's analytic hierarchy process. Manage. Sci. 33 (1987) 1383-1403.

[23] E. Hinloopen, P. Nijkamp and P. Rietveld, The regime method: a new multicriteria technique. Essays and Surveys on Multiple Criteria Decision Making. In: Vol. 209 of Lecture Notes in Economics and Mathematical Systems. Springer, Berlin, Heidelberg (1983) 146-155.

[24] C.L. Hwang, Y.J. Lai and T.Y. Liu, A new approach for multiple objective decision making. Comput. Oper. Res. 20 (1993) 889-899.

[25] H.C. Liu, X.J. Fan, P. Li and Y.Z. Chen, Evaluating the risk of failure modes with extended MULTIMOORA method under fuzzy environment. Eng. App. Artif. Intell. 34 (2014) 168-177.

[26] A. Mardani, A. Jusoh, K. Nor, Z. Khalifah, N. Zakwan and A. Valipour, Multiple criteria decision-making techniques and their applications - a review of the literature from 2000 to 2014. Economic Research-Ekonomska Istraživanja 28 (2015) $516-571$.

[27] R.T. Marler and J.S. Arora, The weighted sum method for multi-objective optimization: new insights. Struct. Multi. Optim. 41 (2010) 853-862.

[28] G. Munda, A NAIADE based approach for sustainability benchmarking. Int. J. Environ. Technol. Manage. 6 (2006) 65-78.

[29] E.E. Noble and P.P. Sanchez, A note on the information content of a consistent pairwise comparison judgment matrix of an AHP decision maker. Theory Decis. 34 (1993) 99-108.

[30] S. Rezaian and S.A. Jozi, Health-safety and environmental risk assessment of refineries using of multi criteria decision making method. Apcbee Proc. 3 (2012) 235-238.

[31] T.L. Saaty, Scaling method for priorities in hierarchical structures. J. Math. Psychol. 15 (1977) $234-281$.

[32] T.L. Saaty, The Analytical Hierarchy Process: Planning, Priority Setting, Resource Allocation. McGraw-Hill (1980).

[33] T.L. Saaty, Decision making with the analytic hierarchy process. Int. J. Serv. Sci. 1 (2008) 83-98.

[34] T.L. Saaty, Decision Making with Dependence and Feedback: The Analytic Network Process: The Organization and Prioritization of Complexity. RWS Publications, Pittsburgh (1996).

[35] V. Sangiorgio, G. Uva and F. Fatiguso, Optimized AHP to overcome limits in weight calculation: a building performance application. J. Constr. Eng. Manage. 144 (2017) 04017101.

[36] V. Sangiorgio, G. Uva and F. Fatiguso, User reporting-based semeiotic assessment of existing building stock at the regional scale. J. Perform. Constr. Facil. 32 (2018) 04018079.

[37] V. Sangiorgio, G. Uva, F. Fatiguso and J.M. Adam, A new index to evaluate exposure and potential damage to RC building structures in coastal areas. Eng. Fail. Anal. 100 (2019) 439-455.

[38] H.S. Shih, H.J. Shyur and E.S. Lee, An extension of TOPSIS for group decision making. Math. Comput. Model. 45 (2007) $801-813$.

[39] G.H. Tzeng and J.J. Huang, Multiple Attribute Decision Making: Methods and Applications. Chapman and Hall/CRC (2011).

[40] M. Velasquez and P.T. Hester, An analysis of multi-criteria decision making methods. Int. J. Oper. Res. 10 (2013) 56-66.

[41] Y.M. Wang, On lexicographic goal programming method for generating weights from inconsistent interval comparison matrices. Appl. Math. Comput. 173 (2006) 985-991.

[42] R. Wang, Z. Zhou, H. Ishibuchi, T. Liao and T. Zhang, Localized weighted sum method for many-objective optimization. IEEE Trans. Evol. Comput. 22 (2016) 3-18.

[43] G. Xie, Modeling decision processes of a green supply chain with regulation on energy saving level. Comput. Oper. Res. $\mathbf{5 4}$ (2015) 266-273. 